\title{
Tumor Necrosis Factor Primes and Metal Particles Activate the NLRP3 Inflammasome in Human Primary Macrophages
}

\section{Jämsen, Eemeli}

2020-05

Jämsen , E , Pajarinen , J , Kouri , V-P , Rahikkala , A, Goodman , S B , Manninen , M , Nordström , D C , Eklund , K K \& Nurmi , K 2020 , ' Tumor Necrosis Factor Primes and Metal Particles Activate the NLRP3 Inflammasome in Human Primary Macrophages ' , Acta Biomaterialia , vol. 108 , pp. 347-357 . https://doi.org/10.1016/j.actbio.2020.03.017

http://hdl.handle.net/10138/328098

https://doi.org/10.1016/j.actbio.2020.03.017

cc_by_nc_nd

acceptedVersion

Downloaded from Helda, University of Helsinki institutional repository.

This is an electronic reprint of the original article.

This reprint may differ from the original in pagination and typographic detail.

Please cite the original version. 


\section{Journal Pre-proof}

Tumor Necrosis Factor Primes and Metal Particles Activate the NLRP3 Inflammasome in Human Primary Macrophages

Eemeli Jämsen, Jukka Pajarinen, Vesa-Petteri Kouri, Antti Rahikkala, Stuart B. Goodman, Mikko Manninen , Dan C. Nordström , Kari K. Eklund, Katariina Nurmi

PII:

DOI:

Reference:

To appear in:

Received date:

Revised date:

Accepted date:
S1742-7061(20)30149-5

https://doi.org/10.1016/j.actbio.2020.03.017

ACTBIO 6635

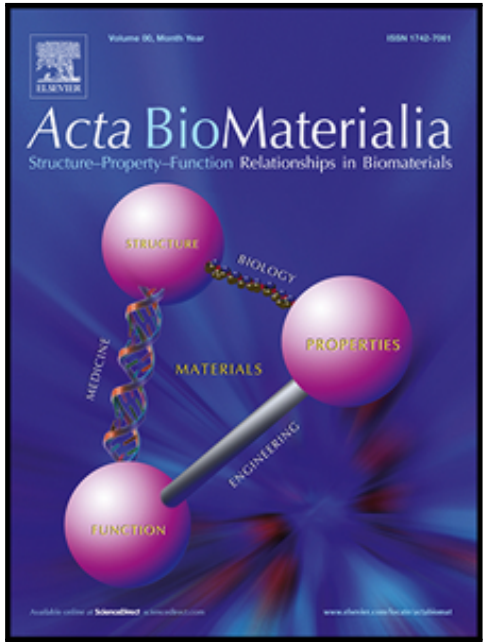

Please cite this article as: Eemeli Jämsen, Jukka Pajarinen, Vesa-Petteri Kouri, Antti Rahikkala, Stuart B. Goodman, Mikko Manninen, Dan C. Nordström, Kari K. Eklund, Katariina Nurmi, Tumor Necrosis Factor Primes and Metal Particles Activate the NLRP3 Inflammasome in Human Primary Macrophages, Acta Biomaterialia (2020), doi: https://doi.org/10.1016/j.actbio.2020.03.017

This is a PDF file of an article that has undergone enhancements after acceptance, such as the addition of a cover page and metadata, and formatting for readability, but it is not yet the definitive version of record. This version will undergo additional copyediting, typesetting and review before it is published in its final form, but we are providing this version to give early visibility of the article. Please note that, during the production process, errors may be discovered which could affect the content, and all legal disclaimers that apply to the journal pertain.

(C) 2020 Acta Materialia Inc. Published by Elsevier Ltd. All rights reserved. 


\section{Tumor Necrosis Factor Primes and Metal Particles Activate the NLRP3 Inflammasome in Human Primary Macrophages}

Eemeli Jämsen ${ }^{a, b, c}$, Jukka Pajarinen ${ }^{a, b, c}$, Vesa-Petteri Kouri, ${ }^{a, b, c}$, Antti Rahikkala ${ }^{d}$, Stuart B.

Goodman $^{\mathrm{e}, \mathrm{f}}$, Mikko Manninen ${ }^{\mathrm{c}}$, Dan C. Nordström ${ }^{\mathrm{a}, \mathrm{b}, \mathrm{c}, \mathrm{g}}$, Kari K. Eklund ${ }^{\mathrm{a}, \mathrm{b}, \mathrm{c}, \mathrm{h}}$, Katariina Nurmi ${ }^{\mathrm{a}, \mathrm{b}, \mathrm{c}}$

a) Department of Medicine, Clinicum, University of Helsinki and Helsinki University Hospital, Haartmaninkatu 8, 00290 Helsinki, Finland

b) Translational Immunology Research Program, University of Helsinki, Haartmaninkatu 8, 00290 Helsinki, Finland

c) ORTON Orthopaedic Hospital of the Orton Foundation, Tenholantie 10, 00280 Helsinki, Finland

d) Division of Pharmaceutical Chemistry and Technology, Faculty of Pharmacy, Viikinkaari $5 \mathrm{E}$, P.O. Box 56, Fl-00014 University of Helsinki, Helsinki, Finland

e) Orthopaedic Research Laboratories, Department of Orthopaedic Surgery, Stanford University School of Medicine, 300 Pasteur Drive, Stanford, CA 94305-5341, USA

f) Department of Bioengineering, Stanford University, 300 Pasteur Drive, Stanford, CA 943055341, USA

g) Internal Medicine and Rehabilitation, University of Helsinki and Helsinki University Hospital, Haartmaninkatu 8, 00290 Helsinki, Finland

h) University of Helsinki and Helsinki University Hospital, Rheumatology, Haartmaninkatu 8, 00290 Helsinki, Finland

Please address correspondence to:

Eemeli Jämsen MD

Department of Medicine Clinicum, University of Helsinki and Helsinki University Hospital

Biomedicum Helsinki 1 , $4^{\text {th }}$ floor, room C403b

Haartmaninkatu 8

00290 Helsinki, Finland

E-mail: eemeli.jamsen@helsinki.fi

Fax: +358-9-471-71897

$\mathrm{T}:+358-29-412-5237$

\section{Abstract}

Aseptic loosening of total joint replacements is driven by a macrophage-mediated inflammatory reaction to implant-derived wear particles. Phagocytosis of implant debris has been suggested to activate the NLRP3 inflammasome leading to secretion of interleukin (IL)-1ß. However, factors and molecular mechanisms driving the particle-induced inflammasome activation are yet to be fully 
elucidated. In this study, we investigated the inflammasome response of human primary macrophages to titanium, chromium, and molybdenum particles in vitro. We observed that particles alone were not sufficient to induce IL-1 $\beta$ secretion, but an additional priming signal—such as bacterial lipopolysaccharide (LPS)—was required to license the inflammasome activation. By using specific inhibitors against the inflammasome signaling pathway, we demonstrate that the particleinduced IL-1 $1 \beta$ secretion depended upon activation of the NLRP3 inflammasome. We further hypothesized that tumor necrosis factor (TNF) could substitute for LPS as a priming signal, and found that particle stimulation together with preceding TNF treatment resulted in inflammasomedependent IL-1 $\beta$ production as well. Our results show that the NLRP3 inflammasome mediates wear particle responses in human primary macrophages, and its activation does not necessarily require the presence of bacterial components, but can be induced under aseptic conditions by TNF priming.

Key words: Macrophage; Wear particle; Inflammasome; IL-1 $\beta$; TNF

\section{Introduction}

Aseptic loosening remains a major long-term complication of total joint replacements and is expected to affect an increasing number of patients [1]. This process, primarily driven by implantderived wear particles, results in a slowly progressive inflammatory osteolysis, jeopardizing the stability of the prosthesis [2]. Due to ongoing wear between implant components, particles are continuously generated and dispersed in the periprosthetic tissues. Consequently, accumulating particulate debris activates macrophages of the innate immune system and the debris becomes engulfed by these phagocytes [3]. To eliminate any potentially dangerous substances and recruit further immune cells to the insult site, macrophages secrete multiple inflammatory mediators $[4,5]$. Despite an intense immune response, wear particles endure the enzymatic degradation of macrophages and sustain the inflammatory stimulus [6]. As a result, macrophage-mediated inflammation develops into a chronic foreign body reaction invading the bone-implant interface [7]. Eventually, this condition increases local bone resorption over bone formation and leads to loosening of the implant. 
Although the established model of aseptic loosening is widely accepted, the exact mechanisms of wear particle-induced macrophage activation remain obscure. Some foreign body particulate materials, such as asbestos and silica, have been shown to activate the NLRP3 (NLR family pyrin domain containing 3 ) inflammasome-a large intracellular machinery mediating the activation of interleukin (IL)-1 $[8,9]$. Indeed, IL-1 $\beta$ represents one of the most potent proinflammatory cytokines and has been identified as a product of wear particle-stimulated macrophages $[5,10]$. Since IL-1 $\beta$ promotes osteoclast function as well, IL-1 $\beta$ is considered a key cytokine in the pathogenetic cascade of aseptic loosening [11]. However, few studies have characterized the underlying mechanisms of NLRP3 inflammasome activation leading to wear particle-induced IL-1 $\beta$ secretion [12-15]. Furthermore, these studies have been conducted primarily with murine macrophages or cell lines.

$\mathrm{IL}-1 \beta$ is first synthesized as a precursor protein (pro-IL-1 $\beta$ ) with the production of biologically active IL-1 $\beta$ strictly regulated by inflammasomes [16]. Among these, the NLRP3 inflammasome is the most versatile [16]. The cytosolic NLRP3 protein belongs to the NLR (nucleotide-binding oligomerization domain-like receptor or NOD) family of pattern recognition receptors capable of sensing various intracellular aberrations such as ion flows, mitochondrial dysfunction, or phagosome rupture [17]. These physiological alterations may result from a diverse array of endo- or exogenous stressors-reportedly also from phagocytosed biomaterial wear particles [12-15]. Upon activation, NLRP3 triggers the assembly of the multimeric inflammasome complex. Subsequent interactions between recruited adaptor proteins ASC (apoptosis-associated speck-like protein containing a caspase-recruitment domain) and pro-caspase-1 lead to autocleavage and formation of active caspase-1. Ultimately, this proteolytic enzyme cleaves precursor protein pro-IL-1 $\beta$ into the mature secreted form.

General consensus agrees that activation of the inflammasome requires two distinct signals [18]. In addition to the actual inflammasome-activating signal detected by NLRP3, a nuclear factor-KB (NF-KB) activating priming signal is needed. This NF-kB-mediated priming licenses the NLRP3 inflammasome by initiating the synthesis of pro-IL-1 $\beta$ and NLRP3 itself. Only together can these two signals activate the inflammasome assembly and induce IL-1 $1 \beta$ secretion from 
macrophages. To date, it remains uncertain whether wear particles alone can provide both of these signals, or whether an additional NF-KB activating priming signal is required. Bacterial lipopolysaccharide (LPS) has been suggested to work in tandem with particles to activate the NF$\mathrm{KB}$ signaling thus licensing the inflammasome, but the amount of LPS in the aseptic interface remains controversial [19-21]. Moreover, the inflammasome-activating potential of different prosthesis materials remains unexamined in a uniform study setting.

In the present study, we investigated the ability of titanium (Ti), chromium (Cr), and molybdenum (Mo) particles to activate the NLRP3 inflammasome in human primary macrophages. The inflammasome activation was assessed in vitro by using qRT-PCR and Western blot analyses, and by measuring the production of IL-1 $\beta$ from culture media with ELISA. We hypothesized that IL$1 \beta$ secretion would depend upon a co-stimulatory priming signal and different events related to activation of the NLRP3 inflammasome. We further asked whether tumor necrosis factor (TNF) could replace LPS as a priming signal and license macrophages for the particle-induced inflammasome activation.

\section{Materials \& Methods}

\subsection{Particle sterilization}

Commercially available particles of common implant materials were purchased from Alfa Aesar (Titanium powder, Product No. 00681; Chromium powder, Product No. 41797; Molybdenum powder, Product No. 10030; Alfa Aesar, Ward Hill, MA). Titanium particles were sterilized with five alternating treatments of $0.1 \mathrm{~N} \mathrm{NaOH}$ in $95 \%$ ethanol and $25 \%$ nitric acid as introduced by Ragab et al [22]. Chromium and molybdenum particles were cleaned with three overnight washes in $70 \%$ ethanol followed by heat sterilization in $175^{\circ} \mathrm{C}$ oven for 3 hours. Particles were suspended in sterile water, and their endotoxin decontamination was verified using EndoLISA (detection limit 0.05 endotoxin units (EU)/mL; ELISA-based Endotoxin Detection Assay, Hyglos, Bernried am Starnberger See, Germany), a limulus amebocyte lysate (LAL) assay kit (detection limit $0.1 \mathrm{EU} / \mathrm{mL}$; Pierce LAL Chromogenic Endotoxin Quantitation Kit, Waltham, MA) and HEK-Blue hTLR4 Cells (InvivoGen, San Diego, CA). These Toll-like receptor (TLR) 4 reporter cells were cultured in DMEM 
medium (Gibco, Waltham, MA) supplemented with 10\% fetal bovine serum (FBS, Gibco) and 1\% Penicillin-Streptomycin antibiotic solution (Gibco), and exposed to particles for 24 hours.

Proportional to TLR4 activation, production of secreted embryonic alkaline phosphatase (SEAP) was assessed from the culture medium with QUANTI-Blue detection reagent (InvivoGen) following the manufacturer's instructions.

For endotoxin detection, particles were analyzed at concentrations corresponding to doses used in cell culture: $2.3 \mathrm{mg} / \mathrm{mL}$ for Ti, $3.6 \mathrm{mg} / \mathrm{mL}$ for $\mathrm{Cr}$, and $5.1 \mathrm{mg} / \mathrm{mL}$ for Mo. LPS levels of the particle solutions remained below the detection limits of EndoLISA and LAL assays, and particle-challenged HEK-Blue cells indicated an SEAP activity comparable to untreated controls. Using the LAL assay, particles were also measured with LPS (from Escherichia coli O111:B4, Sigma, Saint Louis, MO) spikes resulting in recovery rates of $100 \%$ for $\mathrm{Ti}, 27 \%$ for $\mathrm{Cr}$, and $16 \%$ for Mo. Proper function of TLR4 reporter cells was verified using ultrapure LPS (logarithmic standard curve starting from 0.1 EU/mL; from E. coli O111:B4, InvivoGen) and TLR4 inhibitor CLI-095 (1 $\mu \mathrm{g} / \mathrm{mL}$, InvivoGen), and as studied with particles, recovery of LPS (InvivoGen) spikes was $99 \%$ for $\mathrm{Ti}, 73 \%$ for $\mathrm{Cr}$, and $86 \%$ for Mo.

\subsection{Transmission electron microscopy (TEM)}

Particle size and shape were assessed by TEM. 5- $\mu$ l droplets of the metal particle dispersions were pipetted on formvar/carbon 200 mesh grids (FCF200-CU, Electron Microscopy Sciences, Hatfield, PA), and dried by removing excess water using a filter paper (Whatman, Little Chalfont, UK). TEM imaging was performed using a transmission electron microscope (JEM-1400, JEOL, Tokyo, Japan) operated at $80 \mathrm{keV}$ acceleration voltage in bright-field mode.

\subsection{Monocyte isolation and macrophage differentiation}

Human peripheral blood mononuclear cells (PBMCs) were isolated from buffy coats of healthy volunteers, who had signed an informed consent. Buffy coats were by-products of blood preparation intended for clinical use, and their allocation for scientific purposes was approved by the Finnish Red Cross Blood Service (Helsinki, Finland). Blood donors comprised of 30 women 
and 15 men with a mean age of 33 years (range 18-64) for females and 42 years (range 21-67) for males. As qualified for blood donation, donors had no major illnesses or medications.

According to a previously introduced protocol [23], buffy coat blood was diluted in $\mathrm{Ca} / \mathrm{Mg}$-free phosphate-buffered saline (PBS), added on top of Ficoll-Paque PLUS (GE Healthcare, Chicago, IL) density gradient medium, and centrifuged to form a PBMC layer. Mononuclear cells were collected and repeatedly washed with PBS for four times. After the final wash, freshly isolated monocytes were suspended in DMEM medium (Sigma) supplemented with $1 \%$ PenicillinStreptomycin antibiotic solution (Gibco). Cells were counted using a TC20 automated cell counter (Bio-Rad, Hercules, CA) and plated onto 24-well culture plates (Greiner CELLSTAR, Kremsmünster, Austria) at a concentration of $1.5 \times 10^{6}$ cells/well. Cells were allowed to attach for one hour in a humidified $+37^{\circ} \mathrm{C}$ incubator at $5 \% \mathrm{CO}_{2}$, until rinsed twice with PBS to remove nonadherent cells. Attached monocytes were differentiated into macrophages during a seven-day culture in macrophage serum-free medium (macrophage-SFM, Gibco) supplemented with 100 $\mathrm{U} / \mathrm{mL}$ penicillin, $100 \mu \mathrm{g} / \mathrm{mL}$ streptomycin, and $10 \mathrm{ng} / \mathrm{mL}$ granulocyte-macrophage colonystimulating factor (GM-CSF, Miltenyi Biotec, Bergisch Gladbach, Germany).

\subsection{Macrophage stimulation}

Inflammasome priming was induced by culturing macrophages for one hour in the presence of either $1 \mu \mathrm{g} / \mathrm{mL}$ LPS (Sigma) or $100 \mathrm{ng} / \mathrm{mL}$ TNF (R\&D Systems, Minneapolis, MN). Where appropriate, medium of LPS- and TNF-primed macrophages was supplemented for another hour with established inhibitors against NLRP3 inflammasome components or its recognized upstream activating events: leakage of cathepsin B from lysosomes, formation of mitochondrial reactive oxygen species (mtROS), and potassium $\left(\mathrm{K}^{+}\right)$efflux. Two small-molecule inhibitors CY-09 (30 $\mu \mathrm{M}$, Glixx Laboratories Inc, Southborough, MA) and MCC950 (1 $\mu \mathrm{M}$, Avistron, Bude, UK) were used to directly inhibit NLRP3 [24, 25], whereas Z-YVAD-FMK (25 $\mu \mathrm{M}$, Santa Cruz Biotechnology, Dallas, TX) was employed to block the function of caspase-1. Intracellular perturbations sensed by NLRP3 were inhibited using $25 \mu \mathrm{M}$ pan-cathepsin inhibitor CA-074-Me (Calbiochem, San Diego, CA) primarily for cathepsin B, $200 \mu \mathrm{M}$ MitoTEMPO (Sigma) for mtROS, and $130 \mathrm{mM} \mathrm{KCl}$ for $\mathrm{K}^{+}$efflux. 
For the latter, the effect of increased osmolarity was assessed by including a control group treated with $260 \mathrm{mM}$ sorbitol. Working concentrations of LPS and TNF were determined according to previous reports $[23,26]$, whereas the inhibitor concentrations were optimized in our laboratory.

Following the priming step and addition of a specific inhibitor, macrophages were challenged for eight hours with an equal volume of $\mathrm{Ti}, \mathrm{Cr}$, or Mo particles. All experiments were conducted in duplicate wells maintaining the presence of priming and inhibiting agents in the medium with the particles. After particle exposure, culture supernatants were collected, and macrophages were disrupted by adding RLT Plus lysis buffer (Qiagen, Valencia, CA). Cell viability was immediately measured from the media using a lactate dehydrogenase (LDH) detection kit (Roche Diagnostics, Mannheim, Germany). Remaining media and cell lysates were stored at $75^{\circ} \mathrm{C}$ for later use.

An optimal particle concentration for macrophage stimulation was determined in preliminary experiments, in which we assessed particle doses ranging from 0.1 to $0.5 \mathrm{~mm}^{3} /$ well and an exposure time up to 24 hours. Taking into account the dose-dependent inflammatory responses and cytotoxicity, an eight-hour stimulation time with a particle load of $0.25 \mathrm{~mm}^{3} /$ well was chosen for further experiments. In all conditions, phagocytosis of particles was verified by microscopy (Supplementary Video 1).

\section{5 qRT-PCR}

Total RNA (ribonucleic acid) was extracted and purified from cell lysates by using RNeasy Mini Kit (Qiagen) according to the manufacturer's instructions. The amount of RNA was measured with NanoDrop 1000 spectrophotometer (Thermo scientific, Wilmington, DE) and $1 \mu \mathrm{g}$ of RNA from each sample was reverse transcribed into complimentary DNA (cDNA) using iScript cDNA Synthesis Kit (Bio-Rad). Quantitative real-time PCR was performed in LightCycler (Roche) instrument from a reaction mix containing $5 \mathrm{ng}$ of sample cDNA, a pair of human forward and reverse primers (Supplementary Table 1), and HOT FIREPol EvaGreen qPCR SuperMix reagent (Solis BioDyne, Tartu, Estonia). Results were normalized to geometric mean of housekeeping 
genes ribosomal protein lateral stalk subunit P0 (RPLP0), hypoxanthine phosphoribosyltransferase 1 (HPRT1), and beta-2-microglobulin (B2M), and obtained using the comparative Ct method.

\subsection{Enzyme-Linked Immunosorbent Assay (ELISA)}

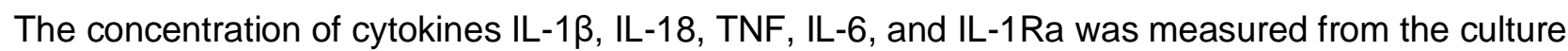
supernatants using ELISA DuoSet kits $(R \& D)$ and high protein binding affinity 96 -well microplates (SpectraPlate-96 HB, Perkin Elmer, Waltham, MA) following the manufacturer's protocol.

\subsection{Western blotting}

For immunoblot analyses, PBMCs were plated onto 6-well culture plates at a concentration of $6 \mathrm{x}$ $10^{6}$ cells/well and differentiated into macrophages as described above. Mature macrophages were primed with $1 \mu \mathrm{g} / \mathrm{mL}$ LPS and challenged with metal particles for eight hours at a concentration of $1.0 \mathrm{~mm}^{3} /$ well. The experiments were run in triplicate wells accompanied by appropriate control groups. Culture media were removed, and adherent macrophages were treated with Cell Lysis Buffer (Cell Signaling Technology, Danvers, MA) containing protease and phosphatase inhibitors (cOmplete Protease Inhibitor Cocktail, Roche; Phosphatase Inhibitor Mini Tablets, Pierce Biotechnology, Waltham, MA). While keeping the plates on ice, cells were scraped, and lysates from triplicate wells were combined. Samples were stored at $-75^{\circ} \mathrm{C}$ for later use.

Cell lysates were thawed, and their protein concentration was measured using a BCA protein assay kit (Pierce) as per the manufacturer's instructions. An equal amount of protein from each sample $(40 \mu \mathrm{g})$ was loaded on gels prepared using TGX FastCast Acrylamide Solutions (BioRad). Proteins were resolved by sodium dodecyl sulfate-polyacrylamide gel electrophoresis (SDSPAGE) and transferred onto a polyvinylidene fluoride (PVDF) membrane (Millipore, Billerrica, MA). The blotted PVDF membranes were blocked with tris-buffered saline containing $0.05 \%$ Tween 20 (TBST) and 5\% milk for one hour at room temperature, after which membranes were incubated overnight at $+4^{\circ} \mathrm{C}$ with antibodies against human IL-1 $\beta$ (1:400, Santa Cruz Biotechnology), NLRP3 (1:1000, AdipoGen, San Diego, CA), and caspase-1 (1:1000, AdipoGen). HRP-conjugated goatanti-mouse or goat-anti-rabbit immunoglobulins were used as secondary antibodies (1:2000, Dako, Glostrup, Denmark). The band intensities were quantified by Bio-Rad ChemiDoc MP imaging 
system and normalized to the intensity of total protein staining on PVDF membrane per lane using stain free technology. This method utilizes polyacrylamide gels containing compounds that modify tryptophan residues in the loaded samples enabling highly sensitive visualization of the protein loading and transfer onto the PVDF membrane [27].

\subsection{Caspase-Glo 1 Assay}

To detect active caspase-1 released in the culture medium, a bioluminescent assay (Caspase-Glo 1 Inflammasome Assay, Promega, Madison, WI) was performed following the manufacturer's protocol. In principle, supernatants were mixed with assay reagent 1:1 and incubated for one hour at room temperature. All conditions were run in duplicates, and the luminescence was measured using a plate-reading luminometer (Tecan, Männedorf, Switzerland).

\subsection{Statistical analyses}

Statistical comparison between the experimental groups was conducted with repeated measures one-way analysis of variance (ANOVA) and Holm-Sidak's post hoc tests using GraphPad Prism version 8 (GraphPad Software, La Jolla, CA). Post hoc analyses were performed separately for each particle material with experimental conditions comprising of at least four independent donors. Paired t-test was used to study statistical significances for TNF-induced pro-IL-1 $\beta$ expression, and for the particle-related secretion of TNF, IL-6, IL-1Ra, and IL-18. P-value of 0.05 was selected as the threshold for significance. Results are presented as mean \pm standard error of mean (SEM).

\section{Results}

\subsection{Characterization of metal particles}

TEM imaging confirmed the particle shape roundish yet irregularly angular (Fig. 1a-c). Analysis of 600 particles revealed an average particle size of $1.6 \pm 1.1 \mu \mathrm{m}$ (range 0.1-7.5 $\mu \mathrm{m}$, median $1.3 \mu \mathrm{m})$ for Ti particles, and a diameter of $1.4 \pm 0.9 \mu \mathrm{m}$ (range 0.2-5.6 $\mu \mathrm{m}$, median $1.0 \mu \mathrm{m}$ ) for Mo particles. Measurement of $400 \mathrm{Cr}$ particles indicated a mean diameter of $1.7 \pm 1.0 \mu \mathrm{m}$ (range 0.3-7.2 $\mu \mathrm{m}$, median $1.5 \mu \mathrm{m})$. Particles of this size range are phagocytable for macrophages and have been 
used in previous in vitro studies [2]. Similar particles have also been identified in periprosthetic tissues around aseptically loosened total joint replacements [28, 29].

\subsection{Inflammasome priming was required for particle-induced IL-1 $\beta$ production}

Using ELISA, we detected no IL-1 $\beta$ secretion from macrophages challenged with wear particles alone. Instead, particle stimulation together with preceding LPS priming led to significant IL-1 $\beta$ production in a dose-dependent manner (Fig. 2). Increasing concentrations of Ti and Mo particles enhanced the amount of IL-1 $\beta$ secreted, whereas a high dose of $\mathrm{Cr}$ particles diminished the cytokine release. A particle volume of $0.25 \mathrm{~mm}^{3}$ per well was therefore selected for subsequent experiments. LPS stimulation alone induced small and consistent IL-1 $1 \beta$ production as well.

\subsection{LPS priming upregulated the expression of pro-IL-1 $\beta$ and NLRP3}

As studied by qRT-PCR, we found that particles alone initiated neither pro-IL-1 $\beta$ nor NLRP3 expression from macrophages. In contrast, LPS-primed cells increased the messenger RNA (mRNA) expression of these markers with or without the addition of metal particles (Fig. $\mathbf{3 a - b}$ ). This phenomenon was further verified by Western blot analysis: While LPS priming resulted in elevated amounts of pro-IL-1 $\beta$ and NLRP3 proteins, these components could not be detected in macrophages challenged with particles alone (Fig. 3c-d). Interestingly, Mo particles in conjunction with LPS induced higher mRNA expression and protein levels of both pro-IL-1 $\beta$ and NLRP3 when compared to Ti or Cr particles.

\subsection{IL-1 $\beta$ secretion depended upon activation of the NLRP3 inflammasome}

To study the role of inflammasome activation in particle-induced IL-1 $\beta$ secretion, we treated LPSprimed macrophages with different inhibitors of NLRP3 inflammasome pathway. Using ELISA assay, we observed that direct inhibition of NLRP3 by either CY-09 or MCC950 significantly diminished the IL-1 $\beta$ release (Fig. 4a-b). With Cr particles intensifying IL-1 $\beta$ secretion to a higher degree, also the inhibitory effect appeared stronger on Cr-challenged macrophages. A similar response profile was seen when inhibiting caspase-1 or cathepsin B by Z-YVAD-FMK or CA-074Me, respectively; these inhibitors reduced the amount of IL-1 $\beta$ secreted in an equally effective 
manner (Fig. 4c-d). Inhibition of $\mathrm{K}^{+}$efflux by high extracellular $\mathrm{K}^{+}$concentration also suppressed the particle-induced IL-1 $\beta$ production (Fig. 4e). The potential effect of increased osmolarity was evaluated by inclusion of sorbitol control groups; particle-stimulated macrophages maintained higher IL-1 $\beta$ levels in otherwise highly osmotic medium (Fig. 4e). Neutralization of mtROS with mitochondria-targeting anti-oxidant MitoTEMPO only slightly mitigated the amount of IL-1 $\beta$ secreted with no statistical significance observable (Fig. 4f). Macrophages treated with inhibitors alone secreted IL-1 $\beta$ comparable to the level of corresponding control cells (Supplementary Figure 1). Based on LDH release, cytotoxicity induced by particles, priming stimuli, or inhibitors was considered non-significant (Supplementary Figure 2).

\subsection{LPS-primed macrophages increased caspase-1 release after particle stimulation} Inflammasome activation was further confirmed by studying the release of active caspase- 1 from cell culture supernatants. Using a luminescence assay, we observed that LPS-primed macrophages showed an enhanced release of active caspase- 1 in response to metal particles when compared to cells without a priming signal (Fig. 5a). Surprisingly, Cr particles alone induced some degree of caspase- 1 secretion as well. Without LPS priming, however, IL-1 $\beta$ secretion from particle-challenged macrophages remained undetectable (Fig. 5b). Using gel electrophoresis, we observed that neither LPS priming nor particle treatment affected the protein expression of procaspase-1. Regardless, no active caspase-1 could be detected from the cell lysates (data not shown).

\subsection{TNF priming enabled particle-induced NLRP3 activation and IL-1 $\beta$ secretion}

In search of a sterile agent that could replace LPS as a priming signal for particle-induced IL-1 $\beta$ production, we found that TNF priming resulted in enhanced pro-IL-1 $\beta$ and NLRP3 expressions at mRNA level (Fig. 6a-b). Whereas pro-IL-1 $\beta$ expression was elevated still at nine hours after the initiation of TNF priming, NLRP3 expression proved to be transient and attenuated already by the four-hour time point. Together with particle stimulation, an elevated IL-1 $\beta$ secretion became evident (Fig. 6c). This finding occurred for all materials studied with $\mathrm{Cr}$ particles again inducing the 
highest levels of IL-1 $1 \beta$ release. Inhibition of NLRP3 and caspase- 1 reduced the particle-related IL$1 \beta$ secretion also from TNF-primed macrophages (Fig. 6d-e).

\subsection{Metal particles alone elicited secretion of other inflammatory cytokines}

As analyzed by ELISA, we discovered that metal particles were able to initiate the secretion of TNF, IL-6, IL-1Ra, and IL-18 without the need of an additional priming signal (Fig. 7a-d). Interestingly, Mo particles markedly increased the release of TNF and IL-6, whereas IL-18 production was enhanced to the highest degree by Cr particles. LPS priming further intensified the secretion of IL-18, which proved to be inflammasome-mediated as inhibition of NLRP3 decreased the secretion of this pro-inflammatory cytokine (Fig. 7e-f).

\section{Discussion}

Excessive and prolonged IL-1 $\beta$ production has been associated with many chronic inflammatory conditions causing progressive tissue damage [30,31]. This scenario seems to prevail in aseptic loosening as well, due to wear debris activation of the innate immune system. Indeed, IL-1 $\beta$ is considered an essential pro-inflammatory mediator driving osteolysis at the bone-implant interface [5]. However, the underlying cellular mechanisms leading to wear particle-induced IL-1 $\beta$ production remain incompletely understood. Therefore, we challenged human primary macrophages with particles from common implant materials, and characterized their effects on priming and activation of the NLRP3 inflammasome pathway-an established regulator of IL-1 $\beta$ secretion.

We observed that macrophages stimulated with metal particles alone elicited no observable IL-1 $\beta$ secretion. While the production of pro-IL-1 $\beta$ and NLRP3 also remained absent, these findings indicate that none of the particles was able to induce NLRP3 inflammasome priming. These results are in line with two recent studies, in which sterile Ti particles induced neither NF-KB activation nor IL-1 $\beta$ secretion from murine macrophages [32, 33]. At the same time, our results are different from previous reports, in which $\mathrm{Ti}$ and $\mathrm{Cr}$-Mo alloy particles, without an additional priming signal, induced macrophage-mediated IL-1 $\beta$ secretion $[10,12,15]$. While these in vitro studies were performed under different experimental conditions likely contributing to priming of cells, traces of endotoxin contamination on particle surfaces could also be one explanation for this discrepancy. 
Indeed, commonly used endotoxin detection assays are sensitive to inhibition by test sample, and may be interfered with implant particles. This can lead to false negative results and misinterpretation of wear particle responses. Rather poor recovery of LPS spikes with Cr and Mo particles in our LAL assay further demonstrates this issue.

Despite these concerns, our results obtained with macrophages and TLR4 reporting HEK-Blue cells imply that no biologically significant amount of endotoxin remained on particles used in the current study. On the contrary, pretreatment with LPS dramatically enhanced secretion of $\mathrm{IL}-1 \beta$ from macrophages suggesting a co-stimulatory priming step necessary for particleinduced inflammasome activation. Increased expressions of pro-IL-1 $\beta$ and NLRP3 at both mRNA and protein levels verify inflammasome priming by LPS treatment. In fact, bacterial LPS stands as a well-documented TLR4 ligand that primes the inflammasome through activation of transcription factor NF-KB [34], and it is commonly used as a priming agent in the field of inflammasome research. To examine the particle-induced inflammasome activation in more detail, further experiments were performed using LPS as a priming signal.

Whereas macrophage responses to Ti and Mo particles proved to be dosedependent, the highest concentration of $\mathrm{Cr}$ particles reduced the amount of IL-1 $1 \beta$ released. While this result was not due to increased cytotoxicity, it is possible that a negative feedback response restricted the effect of an overwhelming particle load. Given that the particle shape and size were similar between different materials, this discrepancy may also originate from unidentified, chromium-specific physico-chemical factors. Conversely, with the selected dose of $0.25 \mathrm{~mm}^{3} /$ well, Cr particles actualiy induced the most vigorous IL-1 $\beta$ secretion throughout the experiments. Hence, it seems that apart from the particle dose, also the material itself affects the magnitude of inflammasome activation. Regardless, particles of every material substantially increased secretion of IL-1 $\beta$ from LPS-primed macrophages suggesting their potential to provide the actual inflammasome-activating signal.

In subsequent mechanistic studies, we demonstrate that the particle-related IL-1 $\beta$ secretion depended upon activation of the NLRP3 inflammasome. Since NLRP3 inflammasome is activated by structurally and chemically divergent triggers, it has emerged that instead of detecting 
each particular activator separately, NLRP3 receptor in fact responds to common cellular stresssignals caused by these stimuli [17]. NLRP3 activation has been closely linked at least with production of $\mathrm{mtROS}, \mathrm{K}^{+}$efflux, and lysosomal destabilization followed by cathepsin $\mathrm{B}$ release [17, 35]. The latter occurs especially from phagocytosis of particulate matter and has been suggested as a means for implant wear to activate the inflammasome $[15,36]$. As pan-cathepsin inhibition reduced the amount of $\mathrm{IL}-1 \beta$ released, this proposal is further supported by our results. In addition, the current study highlights the importance of NLRP3 itself, $\mathrm{K}^{+}$efflux, and caspase- 1 in particleinduced IL-1 $\beta$ secretion; all of these components were needed for a pronounced activation of the NLRP3 inflammasome cascade (Fig. 8). While also mtROS have been suggested to participate in particle-related inflammatory reaction, their inhibition had no significant effect on IL-1 $\beta$ secretion. These products might be more pertinent regarding non-phagocytable particles larger than $20 \mu \mathrm{m}$ in size [37].

To verify complete inflammasome activation, active caspase-1 was further shown from culture supernatants of particle-challenged macrophages. Indeed, inflammasome activation results in formation of membrane pores that allow not only the secretion of $\mathrm{IL}-1 \beta$, but also the release of the entire NLRP3 inflammasome complex including active caspase-1 [38, 39]. This phenomenon was consistent with the absence of active intracellular caspase-1, and with low levels of cytosolic NLRP3 protein in Cr-challenged macrophages, which eventually induced the strongest inflammasome activation. Compared to stimulation with particles alone, addition of LPS increased formation of active caspase-1 emphasizing once again the relevance of a separate priming signal. After all, inhibition of caspase- 1 effectively decreased IL-1 $1 \beta$ secretion indicating activation and involvement of this enzyme in particle-induced inflammasome activation.

Even though LPS intensifies macrophage responses to wear particles, its role in the pathogenesis of aseptic loosening remains debated. On the one hand, endotoxins may be released into circulation from infection foci or due to increased intestinal permeability [40]. In this case, LPS tends to localize onto foreign surfaces such as joint replacement components or wear particles, and thereby likely contributes to implant loosening [41, 42]. On the other hand, evidence for the presence of LPS remains somewhat inconclusive and it is also possible that endotoxins are 
not required for the development of peri-implant osteolysis. Indeed, although a recent study found endotoxin in periprosthetic tissues, endotoxin levels remained undetectable in majority of the samples collected around aseptically loosened joint replacements [43]. Therefore, we studied whether TNF as an alternative sterile agent could provide an adequate priming signal to license the particle-induced inflammasome activation.

Previous studies have identified TNF capable of priming NLRP3 inflammasome by activating the NF-kB via cytokine receptor-mediated signaling pathway [44, 45]. This proinflammatory cytokine is also produced by particle-challenged macrophages and, importantly, its presence in the aseptic interface tissue has been widely recognized [46-48]. Together with TNF priming, we found that particles were able to induce increased IL $-1 \beta$ secretion from human macrophages in an environment devoid of any bacterial constituents. This TNF-related IL-1 $\beta$ production proved to be NLRP3 inflammasome-dependent as well. In comparison with LPS, TNF led to weaker priming and activation of the inflammasome, which is in line with previous studies and fits the clinical picture of a low-grade peri-implant inflammation slowly leading to osteolysis [26]. These results suggest that LPS may not be necessary for wear particle-mediated inflammasome activation, but also TNF can provide the priming signal required for IL-1 $\beta$ secretion (Fig. 8). Of note, along with inflammasome components NLRP3, ASC, and caspase-1, these two cytokines have been identified from the aseptic interface together-correlating with each other and to the severity of osteolysis [46-50]. These findings further underline the relationship between TNF and IL-1 $\beta$ in wear particle-mediated inflammation.

We acknowledge selected doses of LPS and TNF higher than physiologically relevant. Nevertheless, such doses have been previously used to enable inflammasome priming, and the need to overload certain stimulants is generally admitted as a shortcoming of in vitro studies. Particle concentrations used might also be higher than found in periprosthetic tissues, but these doses were verified non-cytotoxic and optimal for this cell culture model. Moreover, inhibition of NLRP3 inflammasome components and its upstream activating events did not entirely block the particle-induced IL-1 $\beta$ or IL-18 secretion. As complete inhibition is difficult to achieve, these results reflect the compromise between inhibitor efficiency and cytotoxicity due to adverse effects of 
exaggerated inhibitor concentrations. Since several upstream activating events may

simultaneously contribute to inflammasome activation, inhibition of one event at a time can result in incomplete inhibition as well. While both $\mathrm{Cr}$ and Mo particles activated the NLRP3 inflammasome, no direct conclusions can be made for the inflammasome activating potential of CrMo alloy particles. The proposed stimulatory effects of $\mathrm{Co}^{2+}$ and other metal ions, as well as cobalt based implant debris also remain speculative $[51,52]$.

To validate our cell culture model, macrophage responses to metal wear particles were further characterized by analyzing the secretion of inflammatory mediators other than IL-1 $\beta$. In line with previous reports, cytokines of both pro- and anti-inflammatory nature were detected from culture supernatants $[4,5]$. Even though production of pro-inflammatory cytokine IL-18 is associated with inflammasome activation as well [18], this cytokine was observed in culture media already without a co-stimulatory priming signal. Particle-induced secretion of mature IL-18 was likely allowed by ready-made pro-IL-18 mRNA, which unlike IL-1 $\beta$ precursor, is constitutively expressed and does not need a priming signal [53]. Particles also induced secretion of TNF, yet by eight-hour time point, its concentrations remained considerably lower than required for inflammasome priming in our cell culture model.

While TNF secretion would likely be increased over time, a multiplicity of additional macrophage stimulating factors can contribute to TNF production and its effects in vivo. Besides accumulating wear particles, complex tissue signaling at the aseptic interface could further enhance TNF secretion from macrophages and increase their activation in response to TNF. In addition to macrophages, TNF may originate from several other cell types including monocytes, endothelial cells, lymphocytes, and fibroblasts. It is noteworthy that LPS is an extremely potent activator of TNF production as well. Thus, its involvement in both TNF secretion and direct inflammasome priming cannot be ruled out. Indeed, although neither TNF nor particles themselves seem to stimulate TLRs, these receptors have been proposed to play a role in the pathogenesis of aseptic loosening [54]. Thus, besides TNF, LPS or other danger signals might still contribute to inflammasome priming through TLR signaling. Since all these environmental signals could not be modeled in our experimental set up, future studies on this subject are warranted in vivo. 
Taken together, this study shows that the NLRP3 inflammasome mediates metal wear particle responses in human primary macrophages. Metal particles themselves, however, remained insufficient to prime the inflammasome, and an additional priming signal was therefore needed. Importantly, the presence of LPS was not necessarily required, but inflammasome activation could be induced under truly aseptic conditions by TNF priming. These results highlight the role of NLRP3 inflammasome in metal particle-induced inflammation, and provide a concept of TNF-related IL-1 $\beta$ secretion in this context. Regardless of the priming signal, inhibition of the NLRP3 inflammasome activation appears a promising means to prevent development of aseptic loosening.

\section{Acknowledgements}

This work was supported by grants from the Finnish Cultural Foundation, ORTON Orthopaedic Hospital of the ORTON Foundation, Alfred Kordelin Foundation, Finnish Medical Foundation, Päivikki and Sakari Sohlberg Foundation, Orion Research Foundation, and Finnish Research Foundation of Orthopaedics and Traumatology. Templates from Servier Medical Art, licensed under a Creative Commons Attribution 3.0 Unported License, were used in graphical abstract and Figure 8; https://smart.servier.com. University of Helsinki Biostatistics unit is acknowledged for consulting with statistical analyses.

\section{Author contributions}

EJ, JP, VPK, KKE and KN constructed the study design. EJ performed the experiments with assistance from AR and KN. EJ, JP and KN analyzed the data and wrote the paper. All authors participated in interpretation of the results, critical revision of the manuscript, and approval of the final version. DN, KKE and KN supervised the project.

\section{Disclosures}

Authors have no conflicts of interest to disclose.

\section{References}


[1] T. Karachalios, G. Komnos, A. Koutalos, Total hip arthroplasty: Survival and modes of failure, EFORT Open Rev 3(5) (2018) 232-239.

[2] Y. Abu-Amer, I. Darwech, J.C. Clohisy, Aseptic loosening of total joint replacements: mechanisms underlying osteolysis and potential therapies, Arthritis research \& therapy 9 Suppl 1 (2007) S6.

[3] C. Nich, S.B. Goodman, Role of macrophages in the biological reaction to wear debris from joint replacements, J Long Term Eff Med Implants 24(4) (2014) 259-265.

[4] C. Nich, Y. Takakubo, J. Pajarinen, M. Ainola, A. Salem, T. Sillat, A.J. Rao, M. Raska, Y. Tamaki, M. Takagi, Y.T. Konttinen, S.B. Goodman, J. Gallo, Macrophages-Key cells in the response to wear debris from joint replacements, Journal of biomedical materials research. Part A 101(10) (2013) 3033-45.

[5] S. Landgraeber, M. Jager, J.J. Jacobs, N.J. Hallab, The pathology of orthopedic implant failure is mediated by innate immune system cytokines, Mediators of inflammation 2014 (2014) 185150. [6] P.H. Wooley, E.M. Schwarz, Aseptic loosening, Gene therapy 11(4) (2004) 402-7. [7] J. Gallo, S.B. Goodman, Y.T. Konttinen, M. Raska, Particle disease: biologic mechanisms of periprosthetic osteolysis in total hip arthroplasty, Innate immunity 19(2) (2013) 213-24.

[8] C. Dostert, V. Petrilli, R. Van Bruggen, C. Steele, B.T. Mossman, J. Tschopp, Innate immune activation through Nalp3 inflammasome sensing of asbestos and silica, Science (New York, N.Y.) $320(5876)(2008) 674-7$.

[9] E.K. Jo, J.K. Kim, D.M. Shin, C. Sasakawa, Molecular mechanisms regulating NLRP3 inflammasome activation, Cellular \& molecular immunology 13(2) (2016) 148-59.

[10] W.J. Maloney, R.E. James, R.L. Smith, Human macrophage response to retrieved titanium alloy particles in vitro, Clinical orthopaedics and related research (322) (1996) 268-78.

[11] T. Shiratori, Y. Kyumoto-Nakamura, A. Kukita, N. Uehara, J. Zhang, K. Koda, M. Kamiya, T. Badawy, E. Tomoda, X. Xu, T. Yamaza, Y. Urano, K. Koyano, T. Kukita, IL-1beta Induces Pathologically Activated Osteoclasts Bearing Extremely High Levels of Resorbing Activity: A Possible Pathological Subpopulation of Osteoclasts, Accompanied by Suppressed Expression of Kindlin-3 and Talin-1, Journal of immunology (Baltimore, Md. : 1950) 200(1) (2018) 218-228. 
[12] M.S. Caicedo, R. Desai, K. McAllister, A. Reddy, J.J. Jacobs, N.J. Hallab, Soluble and particulate Co-Cr-Mo alloy implant metals activate the inflammasome danger signaling pathway in human macrophages: a novel mechanism for implant debris reactivity, Journal of orthopaedic research : official publication of the Orthopaedic Research Society 27(7) (2009) 847-54.

[13] C.A. St Pierre, M. Chan, Y. Iwakura, D.C. Ayers, E.A. Kurt-Jones, R.W. Finberg, Periprosthetic osteolysis: characterizing the innate immune response to titanium wear-particles, Journal of orthopaedic research : official publication of the Orthopaedic Research Society 28(11) (2010) 1418-24.

[14] L. Burton, D. Paget, N.B. Binder, K. Bohnert, B.J. Nestor, T.P Sculco, L. Santambrogio, F.P. Ross, S.R. Goldring, P.E. Purdue, Orthopedic wear debris mediated inflammatory osteolysis is mediated in part by NALP3 inflammasome activation, Journal of orthopaedic research : official publication of the Orthopaedic Research Society 31(1) (2013) 73-80.

[15] M.S. Caicedo, L. Samelko, K. McAllister, J.J. Jacobs, N.J. Hallab, Increasing both CoCrMoalloy particle size and surface irregularity induces increased macrophage inflammasome activation in vitro potentially through lysosomal destabilization mechanisms, Journal of orthopaedic research : official publication of the Orthopaedic Research Society 31(10) (2013) 1633-42.

[16] F.S. Sutterwala, S. Haasken, S.L. Cassel, Mechanism of NLRP3 inflammasome activation, Annals of the New York Academy of Sciences 1319 (2014) 82-95.

[17] A. Abderrazak, T. Syrovets, D. Couchie, K. El Hadri, B. Friguet, T. Simmet, M. Rouis, NLRP3 inflammasome: from a danger signal sensor to a regulatory node of oxidative stress and inflammatory diseases, Redox biology 4 (2015) 296-307.

[18] Y. He, H. Hara, G. Nunez, Mechanism and Regulation of NLRP3 Inflammasome Activation, Trends in biochemical sciences 41(12) (2016) 1012-1021.

[19] A.U. Daniels, F.H. Barnes, S.J. Charlebois, R.A. Smith, Macrophage cytokine response to particles and lipopolysaccharide in vitro, Journal of biomedical materials research 49(4) (2000) 469-78.

[20] Y. Bi, J.M. Seabold, S.G. Kaar, A.A. Ragab, V.M. Goldberg, J.M. Anderson, E.M. Greenfield, Adherent endotoxin on orthopedic wear particles stimulates cytokine production and osteoclast 
differentiation, Journal of bone and mineral research : the official journal of the American Society for Bone and Mineral Research 16(11) (2001) 2082-91.

[21] C.S. Hoenders, M.C. Harmsen, M.J. van Luyn, The local inflammatory environment and microorganisms in "aseptic" loosening of hip prostheses, J Biomed Mater Res B Appl Biomater 86(1) (2008) 291-301.

[22] A.A. Ragab, R. Van De Motter, S.A. Lavish, V.M. Goldberg, J.T. Ninomiya, C.R. Carlin, E.M. Greenfield, Measurement and removal of adherent endotoxin from titanium particles and implant surfaces, Journal of orthopaedic research : official publication of the Orthopaedic Research Society 17(6) (1999) 803-9.

[23] K. Nurmi, I. Kareinen, J. Virkanen, K. Rajamaki, V.P. Kouri, K. Vaali, A.L. Levonen, N. Fyhrquist, S. Matikainen, P.T. Kovanen, K.K. Eklund, Hemin and Cobalt Protoporphyrin Inhibit NLRP3 Inflammasome Activation by Enhancing Autophagy: A Novel Mechanism of Inflammasome Regulation, Journal of innate immunity 9(1) (2017) 65-82.

[24] R.C. Coll, A.A. Robertson, J.J. Chae, S.C. Higgins, R. Munoz-Planillo, M.C. Inserra, I. Vetter, L.S. Dungan, B.G. Monks, A. Stutz, D.E. Croker, M.S. Butler, M. Haneklaus, C.E. Sutton, G. Nunez, E. Latz, D.L. Kastner, K.H. Mills, S.L. Masters, K. Schroder, M.A. Cooper, L.A. O'Neill, A small-molecule inhibitor of the NLRP3 inflammasome for the treatment of inflammatory diseases, Nature medicine 21(3) (2015) 248-55.

[25] H. Jiang, H. He, Y. Chen, W. Huang, J. Cheng, J. Ye, A. Wang, J. Tao, C. Wang, Q. Liu, T. Jin, W. Jiang, X. Deng, R. Zhou, Identification of a selective and direct NLRP3 inhibitor to treat inflammatory disorders, The Journal of experimental medicine 214(11) (2017) 3219-3238. [26] J.S. Bezbradica, R.C. Coll, K. Schroder, Sterile signals generate weaker and delayed macrophage NLRP3 inflammasome responses relative to microbial signals, Cellular \&Amp; Molecular Immunology 14 (2016) 118.

[27] C.P. Moritz, Tubulin or Not Tubulin: Heading Toward Total Protein Staining as Loading Control in Western Blots, Proteomics 17(20) (2017). 
[28] S. Grosse, H.K. Haugland, P. Lilleng, P. Ellison, G. Hallan, P.J. Høl, Wear particles and ions from cemented and uncemented titanium-based hip prostheses-a histological and chemical analysis of retrieval material, J Biomed Mater Res B Appl Biomater 103(3) (2015) 709-717. [29] F. Yang, J. Tang, K. Dai, Y. Huang, Metallic wear debris collected from patients induces apoptosis in rat primary osteoblasts via reactive oxygen speciesmediated mitochondrial dysfunction and endoplasmic reticulum stress, Molecular medicine reports 19(3) (2019) 16291637.

[30] C.A. Dinarello, A clinical perspective of IL-1beta as the gatekeeper of inflammation, European journal of immunology 41(5) (2011) 1203-17.

[31] J.R. Lukens, J.M. Gross, T.-D. Kanneganti, IL-1 family cytokines trigger sterile inflammatory disease, Front Immunol 3 (2012) 315-315.

[32] G.W. Manzano, B.P. Fort, G.R. Dubyak, E.M. Greenfield, Wear Particle-induced Priming of the NLRP3 Inflammasome Depends on Adherent Pathogen-associated Molecular Patterns and Their Cognate Toll-like Receptors: An In Vitro Study, Clinical orthopaedics and related research 476(12) (2018) 2442-2453.

[33] E. Jamsen, J. Pajarinen, T.H. Lin, C.W. Lo, A. Nabeshima, L. Lu, K. Nathan, K.K. Eklund, Z. Yao, S.B. Goodman, Effect of Aging on the Macrophage Response to Titanium Particles, Journal of orthopaedic research : official publication of the Orthopaedic Research Society (2019).

[34] Y.C. Lu, W.C. Yeh, P.S. Ohashi, LPS/TLR4 signal transduction pathway, Cytokine 42(2) (2008) 145-151.

[35] R. Munoz-Planillo, P. Kuffa, G. Martinez-Colon, B.L. Smith, T.M. Rajendiran, G. Nunez, K(+) efflux is the common trigger of NLRP3 inflammasome activation by bacterial toxins and particulate matter, Immunity 38(6) (2013) 1142-53.

[36] V. Hornung, F. Bauernfeind, A. Halle, E.O. Samstad, H. Kono, K.L. Rock, K.A. Fitzgerald, E. Latz, Silica crystals and aluminum salts activate the NALP3 inflammasome through phagosomal destabilization, Nature immunology 9(8) (2008) 847-56.

[37] N. Cobelli, B. Scharf, G.M. Crisi, J. Hardin, L. Santambrogio, Mediators of the inflammatory response to joint replacement devices, Nature reviews. Rheumatology 7(10) (2011) 600-8. 
[38] A. Baroja-Mazo, F. Martin-Sanchez, A.I. Gomez, C.M. Martinez, J. Amores-Iniesta, V.

Compan, M. Barbera-Cremades, J. Yague, E. Ruiz-Ortiz, J. Anton, S. Bujan, I. Couillin, D. Brough, J.I. Arostegui, P. Pelegrin, The NLRP3 inflammasome is released as a particulate danger signal that amplifies the inflammatory response, Nature immunology 15(8) (2014) 738-48.

[39] V.S. Tapia, M.J.D. Daniels, P. Palazón-Riquelme, M. Dewhurst, N.M. Luheshi, J. Rivers-Auty,

J. Green, E. Redondo-Castro, P. Kaldis, G. Lopez-Castejon, D. Brough, The three cytokines IL-1 $\beta$, IL-18, and IL-1 $\alpha$ share related but distinct secretory routes, J Biol Chem 294(21) (2019) 83258335.

[40] H. Fukui, Increased Intestinal Permeability and Decreased Barrier Function: Does It Really Influence the Risk of Inflammation?, Inflamm Intest Dis 1(3) (2016) 135-145.

[41] E.M. Greenfield, Y. Bi, A.A. Ragab, V.M. Goldberg, J.L. Nalepka, J.M. Seabold, Does endotoxin contribute to aseptic loosening of orthopedic implants?, J Biomed Mater Res B Appl Biomater 72(1) (2005) 179-85.

[42] J.L. Nalepka, M.J. Lee, M.J. Kraay, R.E. Marcus, V.M. Goldberg, X. Chen, E.M. Greenfield, Lipopolysaccharide found in aseptic loosening of patients with inflammatory arthritis, Clinical orthopaedics and related research 451 (2006) 229-35.

[43] E.S. Hartmann, M.I. Kohler, F. Huber, J.I. Redeker, B. Schmitt, M. Schmitt-Sody, B. Summer, A. Fottner, V. Jansson, S. Mayer-Wagner, Factors regulating bone remodeling processes in aseptic implant loosening, Journal of orthopaedic research : official publication of the Orthopaedic Research Society 35(2) (2017) 248-257.

[44] F.G. Bauernfeind, G. Horvath, A. Stutz, E.S. Alnemri, K. MacDonald, D. Speert, T. FernandesAlnemri, J. Wu, B.G. Monks, K.A. Fitzgerald, V. Hornung, E. Latz, Cutting edge: NF-kappaB activating pattern recognition and cytokine receptors license NLRP3 inflammasome activation by regulating NLRP3 expression, Journal of immunology (Baltimore, Md. : 1950) 183(2) (2009) 787 91.

[45] L. Franchi, T. Eigenbrod, G. Núñez, Cutting edge: TNF-alpha mediates sensitization to ATP and silica via the NLRP3 inflammasome in the absence of microbial stimulation, Journal of immunology (Baltimore, Md. : 1950) 183(2) (2009) 792-796. 
[46] J. Chiba, H.E. Rubash, K.J. Kim, Y. Iwaki, The characterization of cytokines in the interface tissue obtained from failed cementless total hip arthroplasty with and without femoral osteolysis, Clinical orthopaedics and related research (300) (1994) 304-12.

[47] L.C. Jones, C. Frondoza, D.S. Hungerford, Immunohistochemical evaluation of interface membranes from failed cemented and uncemented acetabular components, Journal of biomedical materials research 48(6) (1999) 889-98.

[48] Y. Naganuma, Y. Takakubo, T. Hirayama, Y. Tamaki, J. Pajarinen, K. Sasaki, S.B. Goodman, M. Takagi, Lipoteichoic acid modulates inflammatory response in macrophages after phagocytosis of titanium particles through Toll-like receptor 2 cascade and inflammasomes, Journal of biomedical materials research. Part A 104(2) (2016) 435-44.

[49] S. Stea, M. Visentin, D. Granchi, G. Ciapetti, M.E. Donati, A. Sudanese, C. Zanotti, A. Toni, Cytokines and osteolysis around total hip prostheses, Cytokine 12(10) (2000) 1575-9.

[50] E. Jamsen, V.P. Kouri, M. Ainola, S.B. Goodman, D.C. Nordstrom, K.K. Eklund, J. Pajarinen, Correlations between macrophage polarizing cytokines, inflammatory mediators, osteoclast activity, and toll-like receptors in tissues around aseptically loosened hip implants, Journal of biomedical materials research. Part A 105(2) (2017) 454-463.

[51] L. Samelko, S. Landgraeber, K. McAllister, J. Jacobs, N.J. Hallab, Cobalt Alloy Implant Debris Induces Inflammation and Bone Loss Primarily through Danger Signaling, Not TLR4 Activation: Implications for DAMP-ening Implant Related Inflammation, PloS one 11(7) (2016) e0160141. [52] M.-A. Ferko, 1. Catelas, Effects of metal ions on caspase-1 activation and interleukin-1 $\beta$ release in murine bone marrow-derived macrophages, PloS one 13(8) (2018) e0199936e0199936.

[53] A.J. Puren, G. Fantuzzi, C.A. Dinarello, Gene expression, synthesis, and secretion of interleukin 18 and interleukin 1 beta are differentially regulated in human blood mononuclear cells and mouse spleen cells, Proc Natl Acad Sci U S A 96(5) (1999) 2256-2261.

[54] M. Takagi, Y. Takakubo, J. Pajarinen, Y. Naganuma, H. Oki, M. Maruyama, S.B. Goodman, Danger of frustrated sensors: Role of Toll-like receptors and NOD-like receptors in aseptic and septic inflammations around total hip replacements, J Orthop Translat 10 (2017) 68-85. 
a) Titanium

$\cdot 8$

$\underline{5 \mu m}$ b)

1
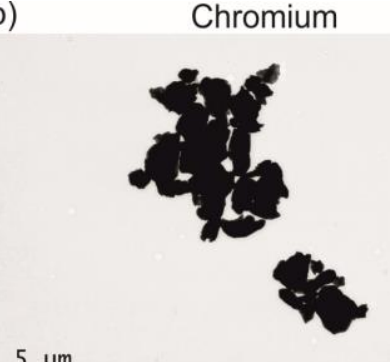

$5 \mu \mathrm{m}$ c)

Molybdenum

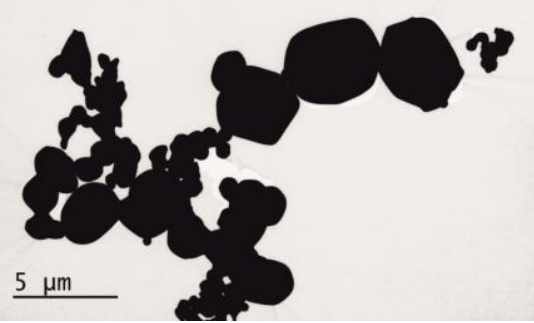

Figure 1. Transmission electron microscopy (TEM) of metal particles. The appearance and sizedistribution of (a) Ti, (b) $\mathrm{Cr}$, and (c) Mo particles were assessed by TEM imaging. Scale bars $5 \mu \mathrm{m}$.

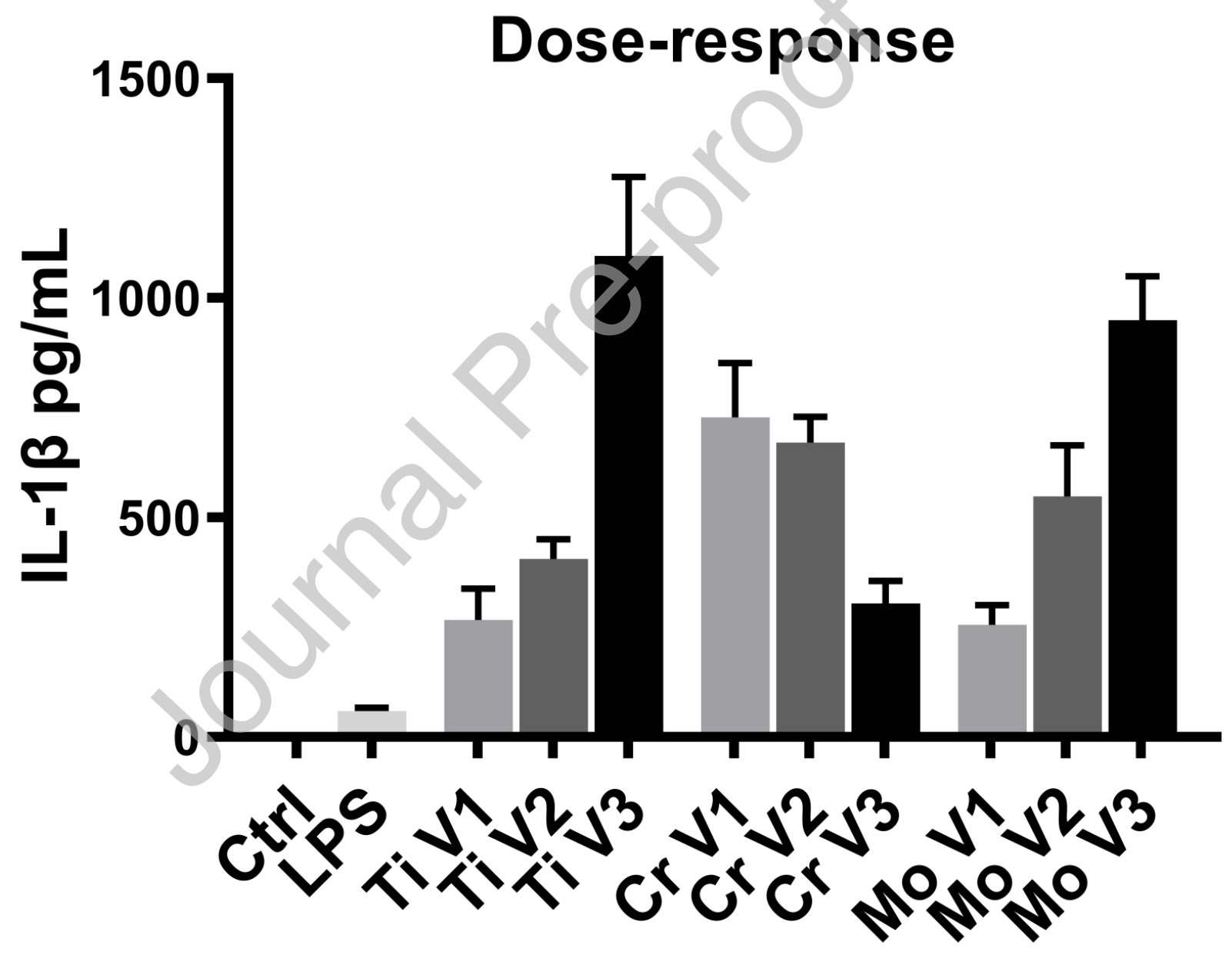

Figure 2. Dose-response of particle-challenged macrophages. Human primary macrophages $(n=$ 6) were primed with LPS and stimulated for eight hours with increasing concentrations of $\mathrm{Ti}, \mathrm{Cr}$, 
and Mo particles. IL-1 $\beta$ secretion induced by particle doses of (V1) 0.1 , (V2) 0.25 , and (V3) 0.5 $\mathrm{mm}^{3} /$ well was determined from culture supernatants by ELISA.

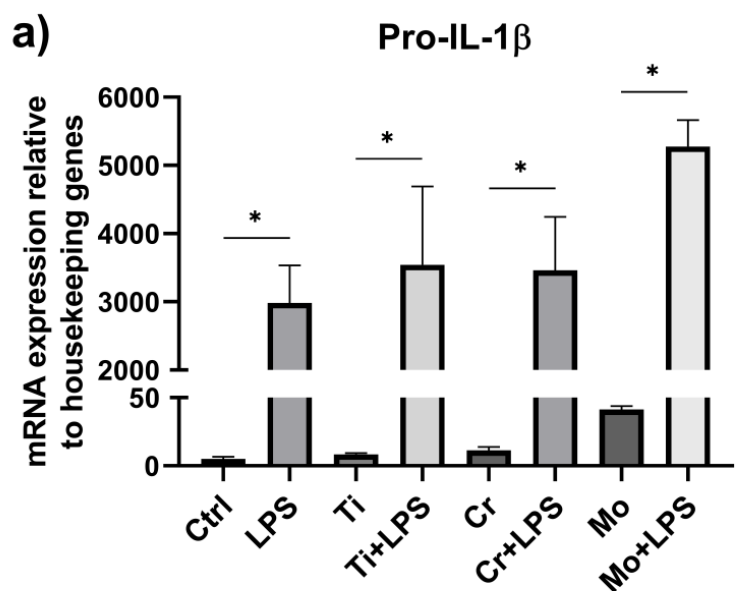

b)

NLRP3

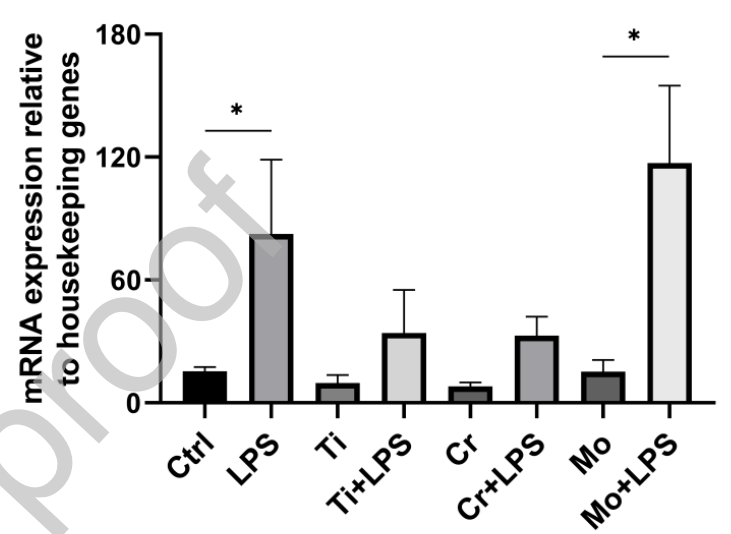

c)

Pro-IL-1 $\beta$

d)

NLRP3
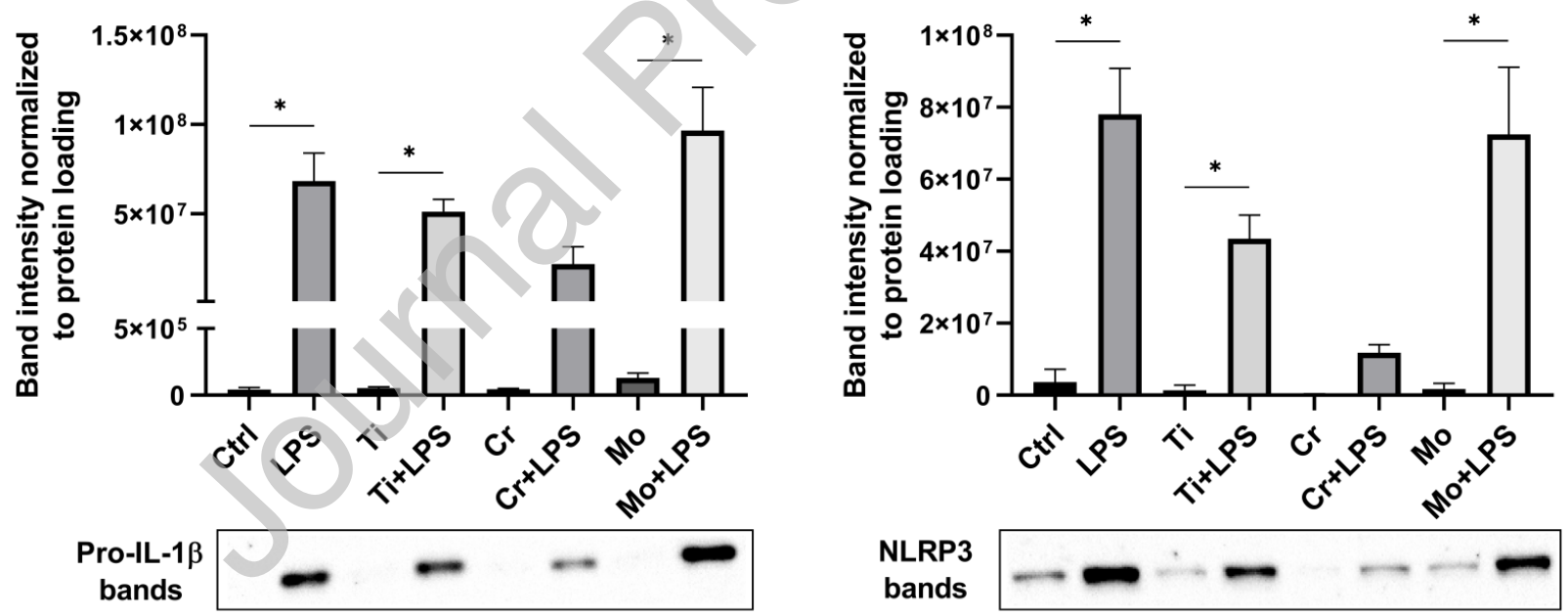

NLRP3

bands

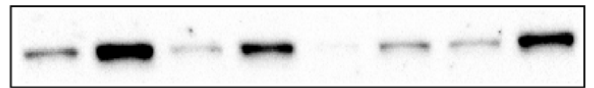

Figure 3. The expression of pro-IL-1 $\beta$ and NLRP3. Priming effects of LPS and particles alone on macrophages $(n=4)$ were analyzed by qRT-PCR and Western blotting. The relative mRNA expression of (a) pro-IL-1 $\beta$ and (b) NLRP3, and the production of intracellular (c) pro-IL-1 $\beta$ and (d) NLRP3 proteins were determined after an eight-hour stimulation with $\mathrm{Ti}, \mathrm{Cr}$, and Mo particles. Representative Western blot images are included with * illustrating statistically significant difference between indicated conditions $(p<0.05)$. 
a) Inhibition of NLRP3

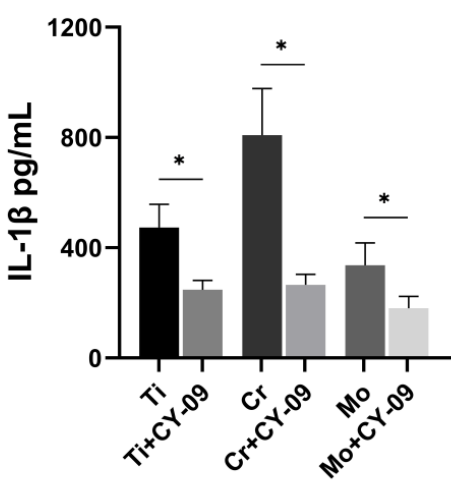

d) Inhibition of cathepsin B

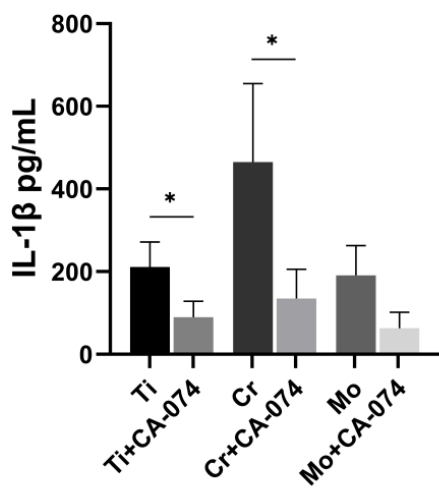

b)

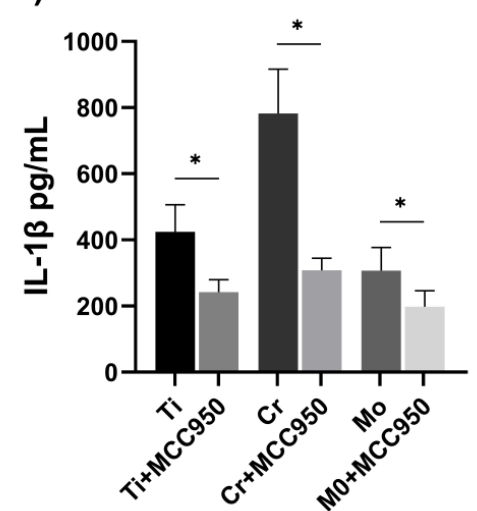

e) Inhibition of $\mathrm{K}^{+}$efflux

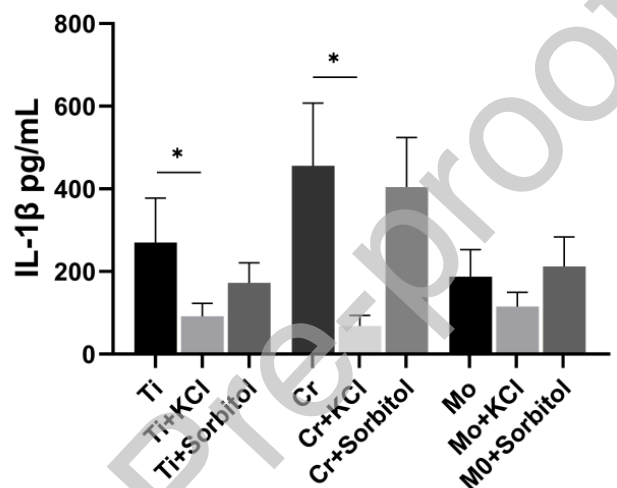

C) Inhibition of caspase-1

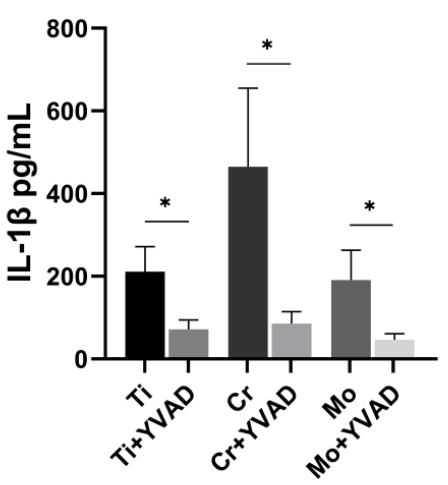

f) Inhibition of mtROS

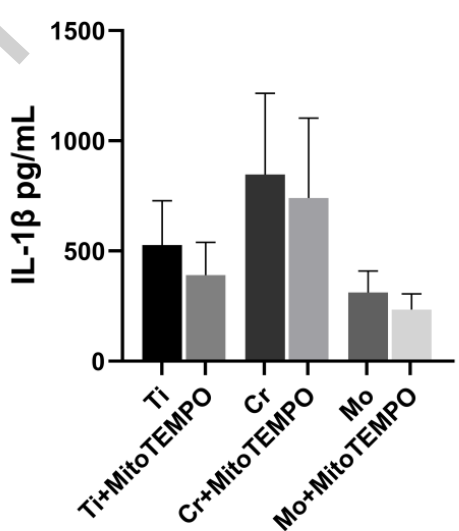

Figure 5. Activation of caspase-1. Human primary macrophages $(n=5)$ were primed with LPS and challenged with $\mathrm{Ti}, \mathrm{Cr}$, and Mo particles for eight hours. (a) The release of active caspase- 1 was measured from culture supernatants using a luminescence assay, and (b) the corresponding IL-1 $\beta$ secretion was determined by ELISA. * represents statistical significance between indicated conditions $(p<0.05)$. 

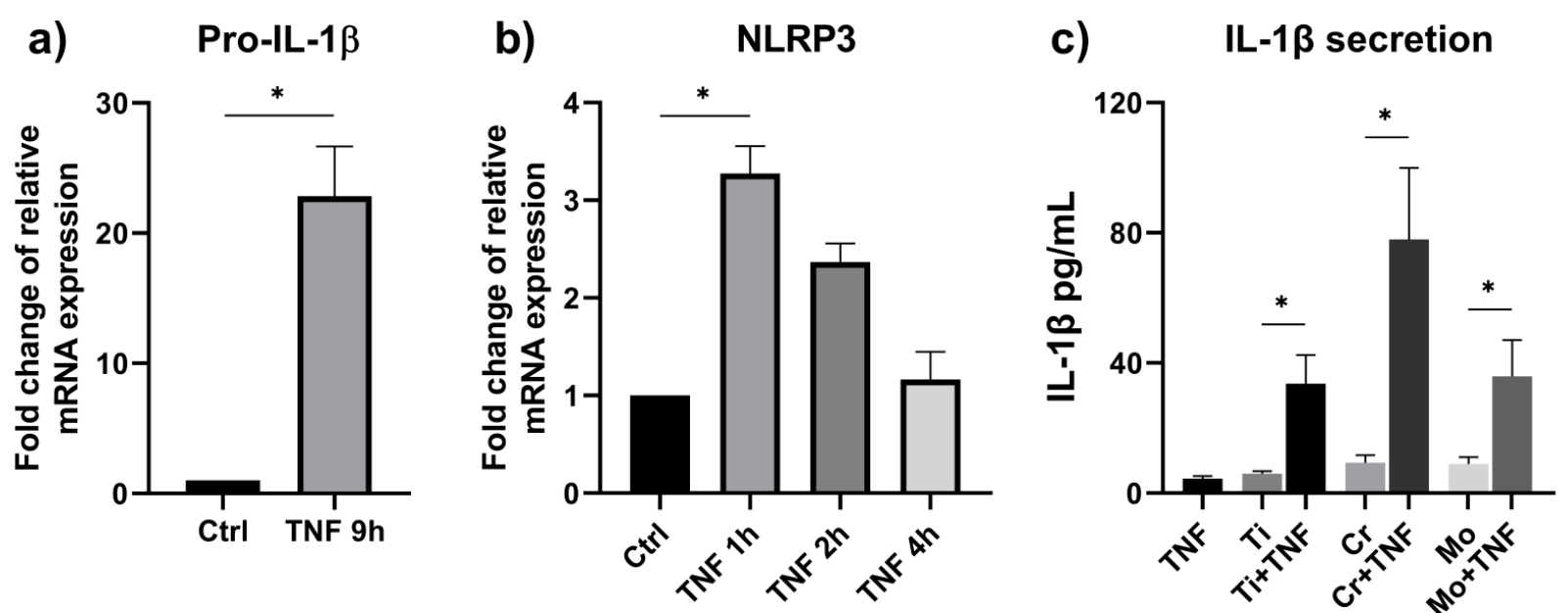

d) Inhibition of NLRP3

e) Inhibition of caspase-1
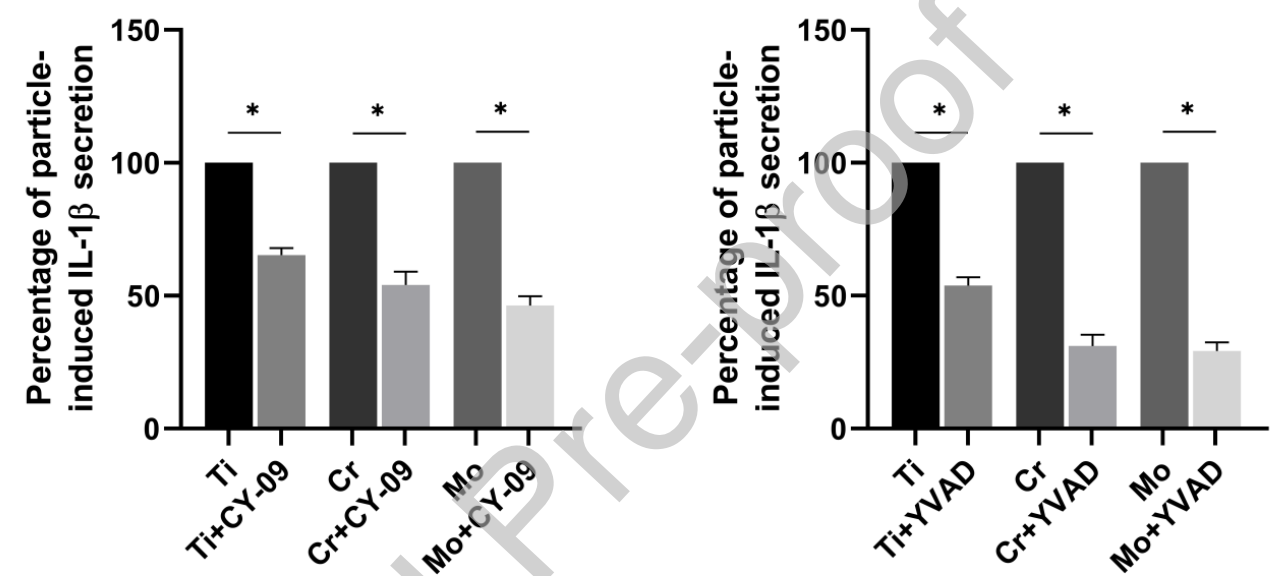

Figure 6. The effect of TNF priming. Human primary macrophages were cultured in the presence of TNF up to nine hours, after which the relative mRNA expression of (a) pro-IL-1ß $(n=3)$ and (b) NLRP3 $(n=4)$ was assessed by qRT-PCR analysis. (c) TNF-primed macrophages $(n=5)$ were exposed to an eight-hour stimulation with $\mathrm{Ti}, \mathrm{Cr}$, and Mo particles, and the IL-1 $\beta$ secretion was assessed by ELISA. (d-e) Particle-induced IL-1 $\beta$ secretion from TNF-primed cells $(n=4)$ was further modified by inhibition of NLRP3 and caspase-1. Data in graphs (a) and (b) are presented as fold change compared to untreated control, whereas results from experiments (d) and (e) are shown as percentage of particle-induced IL-1 $\beta$ secretion. * indicates statistically significant differences between groups $(p<0.05)$. 

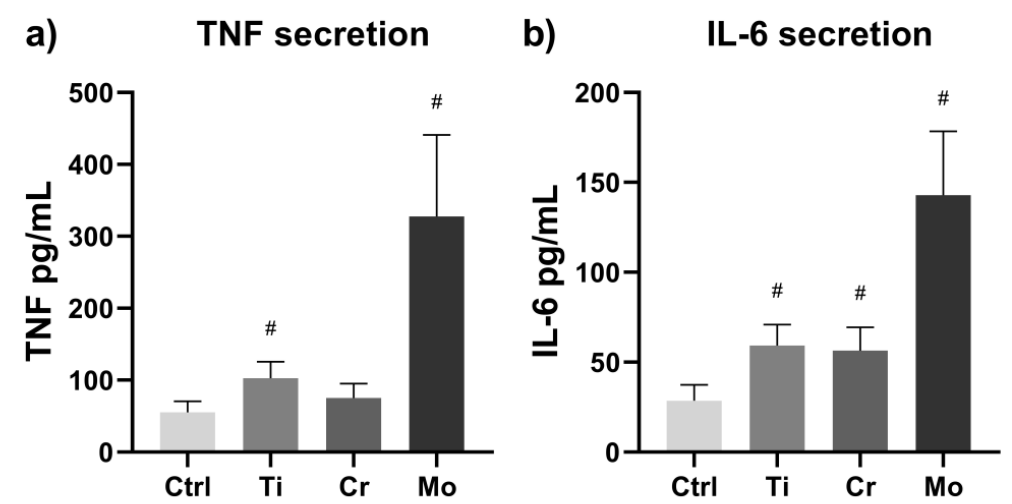

c) IL-1Ra secretion

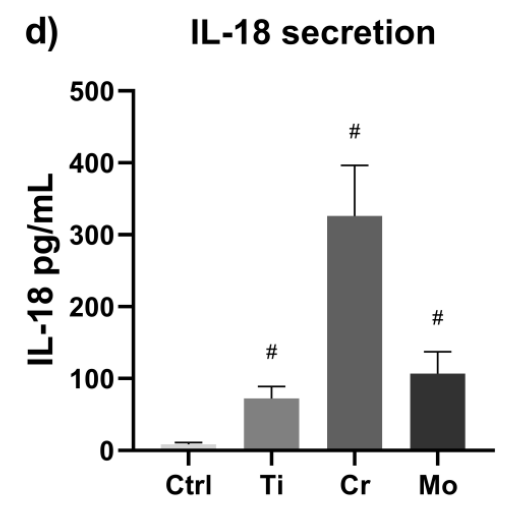

e) Inhibition of NLRP3
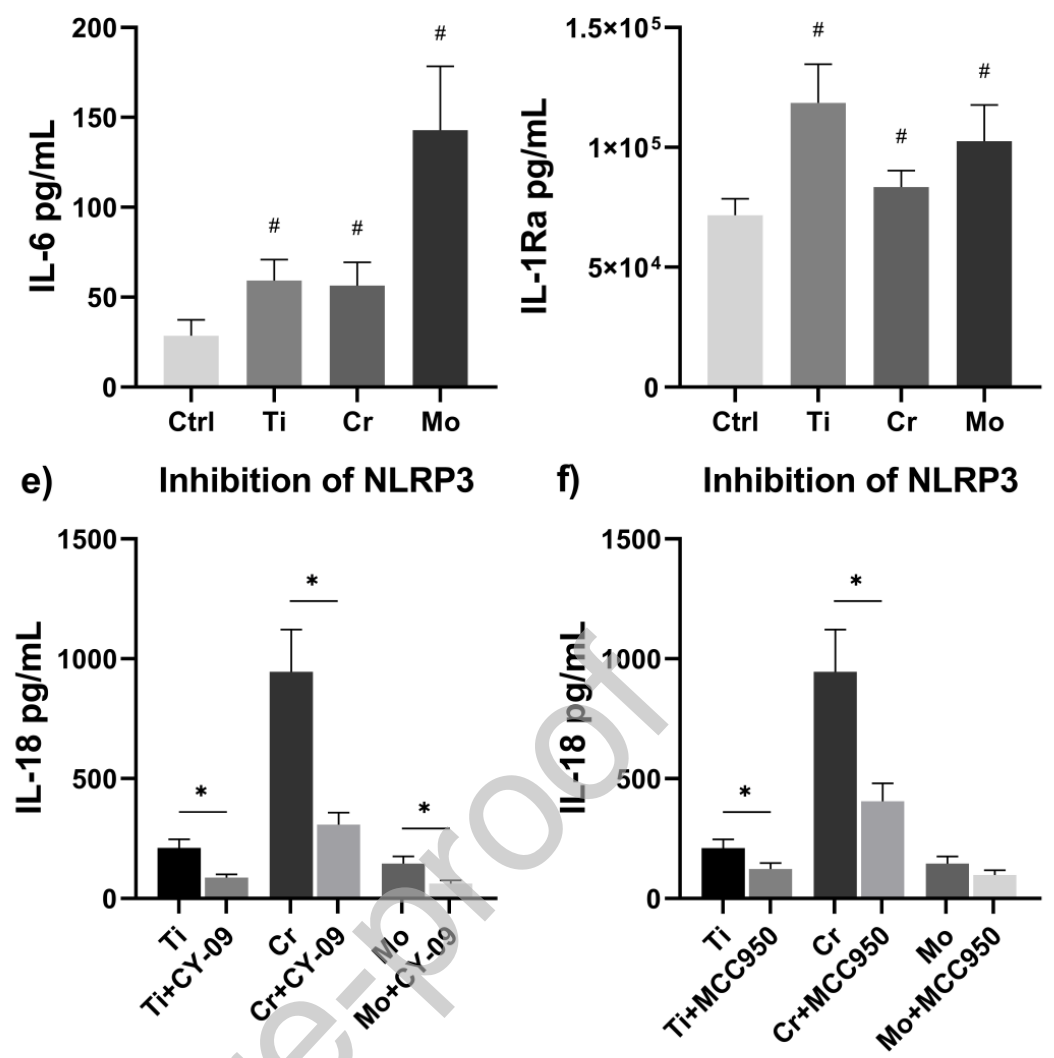

Figure 7. Particle-induced secretion of inflammatory cytokines. Unprimed human primary macrophages were challenged with $\mathrm{Ti}, \mathrm{Cr}$, and Mo particles for eight hours, and the secretion of (a) TNF, (b) IL-6, (c) IL-1Ra, and (d) IL-18 was analyzed from culture supernatants using ELISA assays. LPS-primed macrophages were treated with NLRP3 inhibitors and exposed to an eighthour particle stimulation as well; the inhibitory effects of (e) CY-09 and (f) MCC950 on particleinduced IL-18 secretion were assessed by ELISA. In graphs $(a-c) n=8$, whereas experiments illustrated in graphs (d-f) were performed with $n=4$. \# illustrates statistical significance for particle stimulation, and * represents significant difference between indicated conditions $(p<0.05)$. 
Figure 8.pptx

Figure 8. Schematic view of the wear particle-induced inflammasome activation. 1. An NF-kB activating priming signal such as LPS or TNF is required to synthesize NLRP3 and pro-IL-1 $\beta$ thus licensing macrophages for the NLRP3 inflammasome activation. 2. Recognition of foreign body particulate material results in particle phagocytosis and formation of phagolysosomes. Subsequent intracellular aberrations including 3. leakage of lysosomal protease cathepsin B and 4. outflow of potassium $\left(\mathrm{K}^{+}\right)$are sensed by the NLRP3 receptor. 5. Upon activation, NLRP3 triggers the assembly of the inflammasome complex by recruiting specific adaptor proteins. 6 . NLRP3 inflammasome eventually generates active caspase -1 , which cleaves precursor protein pro-IL-1 $\beta$ into the secreted form. 7. Mature IL-1 $\beta$ is released from the cell through membrane pores formed after the inflammasome activation. 
Graphical abstract.pptx

\section{Graphical Abstract}

